\title{
MULTIPLEX PNEUMATIC CONTROL METHOD FOR MULTI-DRIVE SYSTEM
}

\author{
Yasutaka Nishioka ${ }^{1}$, Koichi Suzumori ${ }^{1}$, Takefumi Kanda ${ }^{1}$, Shuichi Wakimoto ${ }^{2}$
}

${ }^{1}$ Graduate School of Natural Science and Technology, Okayama University, 3-1-1 Tsushima-naka,

kita-ku, Okayama 700-8530, Japan.

${ }^{2}$ Research Core for Interdisciplinary Sciences, Okayama University, 3-1-1 Tsushima-naka, kita-ku,

Okayama 700-8530, Japan.

* Corresponding author, FAX: +81 86251 8159, e-mail: yasu@act.sys.okayama-u.ac.jp 
Abstract

Pneumatic actuators have several advantages such as light weight, safety, low cost and high compliance. However, many pneumatic actuators have complicated systems that include a compressor, air tubes, and pneumatic valves with electrical wires. This research proposes a new control method for a multiplex pneumatic transmission constructed with special resonant valves and air tubes with a control system driven by air vibration in air tubes without electrical wires. The control is simplified and effective for pneumatic systems having many degrees of freedom. In this paper, the development of a primitive model of the resonant valve and a prototype valve is described. In addition, two control methods, which are a superimposing method and a time-sharing method, are shown, and the independent driving of four actuators is realized by using one of the control methods with air tubes only.

Key Words: Pneumatic, Valve, Actuator, Multi-DOF, Resonant, Mechatronics 


\section{Introduction}

Pneumatic actuators are used in several areas, such as industrial robots, rehabilitation tools, and medical and caregiver robots, because they have the advantages of light weight, low cost, safety, and high compliance. However, in a pneumatic system, a large compressor and many electrical wires from the control valve complicate the entire system [1,2].

To solve this problem, new servo controls, including downsized or new pneumatic devices, have been researched [1]. Air compressors which have portability and high capacity have been developed [3, 4]. In addition, downsized pneumatic valves have been proposed [5-8]. For example, a solenoid actuator, electrical motor, and piezoelectric actuator have been adapted to the pneumatic valve. The pneumatic valve using a piezoelectric actuator is suitable for downsizing and improvement of the responsibility $[7,8]$.

The authors previously proposed a novel pneumatic control system called multiplex pneumatic transmission $[9,10]$. The purpose of this system is simplification of the conventional pneumatic system, which is constructed with a many pneumatic solenoid valves and electric wires. Figure 1 shows the proposed system. The multiplex pneumatic transmission is constructed with an oscillator, resonant valves and air tubes. In this system, the resonant valves use air vibration to work as on/off valves. The air vibration is generated by the oscillator, which can be a servo valve, voice coil motor or speaker. In Fig. 1, for example, resonant valve 1 is driven by air vibration 1 having the resonant 
frequency of valve 1 . Similarly, resonant valve 2 is driven by air vibration 2 . By using air vibration 3 combined with the resonant frequencies of valves 1 and 2, both resonant valves 1 and 2 can be driven. Thus, this system simplifies and downsizes the pneumatic valves and the entire system.

In related work, Kitagawa et al. developed a pneumatic pilot valve driven by sound vibration using a speaker [11]. In this research, two pneumatic pilot valves were developed and achieved independent driving of the two valves. This system was adapted to wearable devices in the caretaking and medical assistance areas. Ikuta et al. developed a band pass valve driven by hydraulic pressure for a system with multiple degrees of freedom [12]. Several band pass valves can individually be controlled by different ranges of pressure. When configured with a soft actuator, these valves can be adapted to a safe active catheter having two degrees of freedom.

The authors of the current paper previously defined the working principle behind a proposed pneumatic valve, developed a prototype valve, and experimentally confirmed independent driving of two pneumatic cylinders [13-15].

In this paper, we describe a new resonant valve that successfully realizes independent control of four pneumatic actuators. A new control method of time sharing is proposed for systems having multiple degrees of freedom. In addition, the experimental results of the superimposing method used in the previous research is compared with the time-sharing method. 


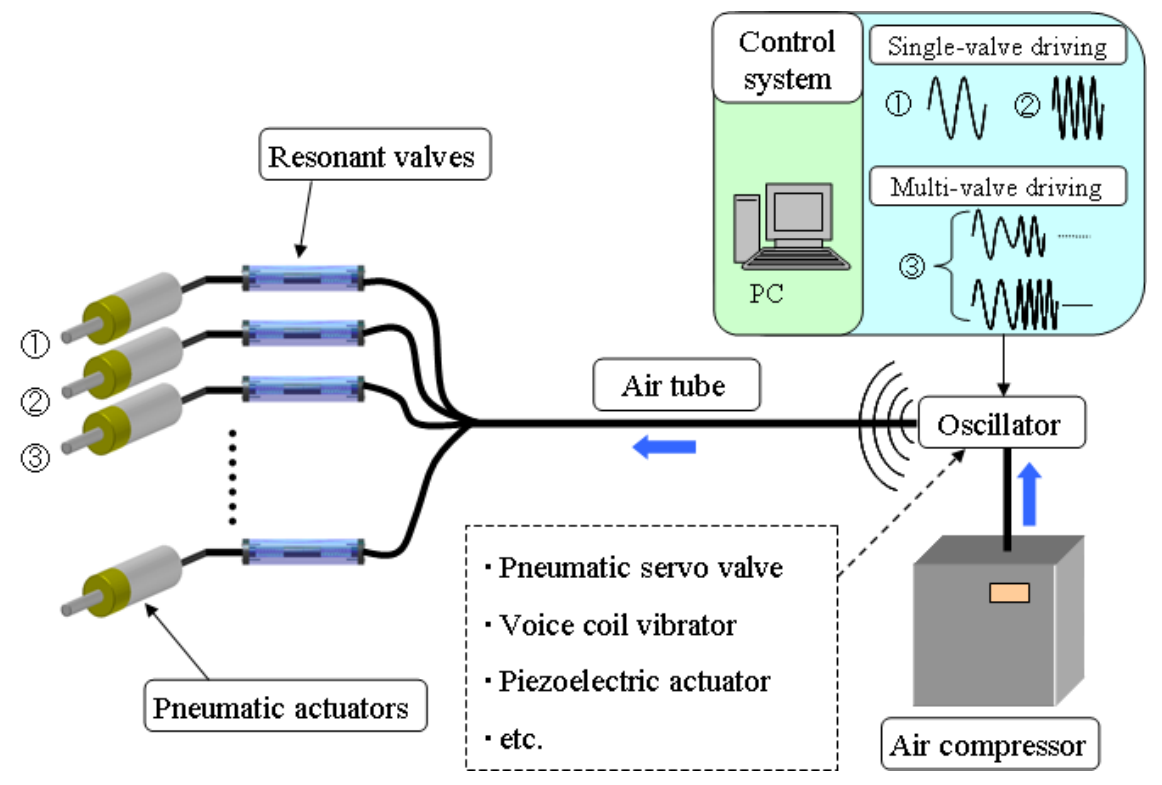

Fig.1 Proposed novel pneumatic system called the multiplex pneumatic transmission $[9,10]$

\section{Working principles of the multiplex pneumatic control system}

\subsection{Working principle of the resonant valve}

The primitive model of the resonant valve is shown in Fig. $2[9,10]$. The model is configured with two mass-spring systems. The vibrators are pressurized between two spring forces and have different channels in each vibrator. In Fig. 2, the input channel of this model is on the left side, and the air vibration from the input channel flows to the right side. When the frequency of the air vibration is the non-resonant frequency, the two objects vibrate on contact. Thus, the air vibration does not flow to the right side. However, when the frequency is the resonant frequency of this model, the two vibrators repeat the contact and non-contact actions between the two vibrating objects. The air vibration flows to the right side through the two vibrating objects. In other words, this model is driven by the air vibration transmitted in air tubes working as an on/off valve. 
The structure of the primitive model was the basis behind the principle figure. The two vibrators and springs were placed in a hollow rectangular case. In the experiment of the primitive model, the oscillator in the multiplex pneumatic transmission was the pneumatic servo valve.

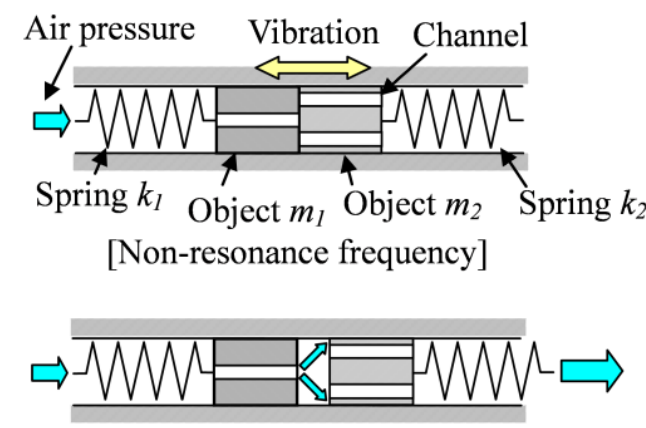

[Resonance frequency]

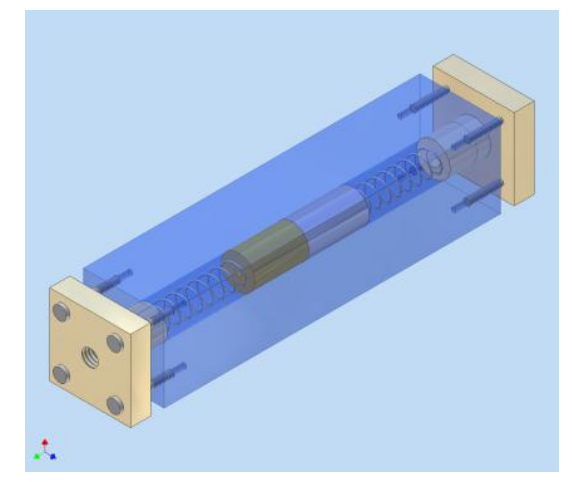

Fig.2 Principle of the primitive model for the resonant valve

\subsection{Superimposing and time-sharing methods}

The resonant valve is controlled by the air vibration of Eq. (1), shown below. Air vibration to the resonant valve is produced by the high-speed valve as the oscillator. In Eq. (1), $V_{p}$ is the voltage parameter to the oscillator and $f_{n}$ is the frequency of the driving valve. Single-valve control is possible by selecting the resonant frequency.

$$
V_{p}=\text { amplitude } \times \sin \left(2.0 \times \pi \times f_{n} \times t\right)+\text { offset }
$$

We propose the superimposing and time-sharing methods of control for driving multiple resonant valves. In the superimposing method, the numbers of frequencies are combined as shown in Fig. 3 
and Eq. (2), where $m$ is the maximum number of resonant valves. Independent control of two

pneumatic cylinders by the superimposing method was successful [13, 14].

$$
V_{p}=\frac{1}{m} \times \sum_{n=1}^{m}\left(\text { amplitude } \times \sin \left(2.0 \times \pi \times f_{n} \times t\right)+\text { offset }\right)
$$

In the time-sharing method, different resonant frequencies are oscillated serially in a short time $T$, as shown in Fig. 4 and Eq. (3).

$$
V_{p}=\left\{\begin{array}{c}
\text { frequency }(1):\left(0 \leq t \leq T_{1}\right) \cdots \text { amplitude } \times \sin \left(2.0 \times \pi \times f_{1} \times t\right)+\text { offset } \\
\text { frequency }(2):\left(T_{1} \leq t \leq T_{2}\right) \cdots \text { amplitude } \times \sin \left(2.0 \times \pi \times f_{2} \times t\right)+\text { offset } \\
\text { frequency }(3):\left(T_{2} \leq t \leq T_{3}\right) \cdots \text { amplitude } \times \sin \left(2.0 \times \pi \times f_{3} \times t\right)+\text { offset } \\
\vdots \\
\text { frequency }(m):\left(T_{m-1} \leq t \leq T_{m}\right) \cdots \text { amplitude } \times \sin \left(2.0 \times \pi \times f_{m} \times t\right)+\text { offset }
\end{array}\right.
$$

Each periods $T_{1}, \cdots, T_{m}$ is decided so that a few waves at least are included in one period. Here, $T_{m}$ is the parameter of the cycle of each resonant frequency. Each frequency is repeated as the cycle is connected serially from frequency (1) to frequency $(m)$. Time $T$ depends on the time constant of the pressure response and wavelength of each resonant frequency. It is effective because the cycle becomes shorter with high-frequency bands. 
In this paper, the number of driving prototype valves is four. The comparison of the two methods is described in an experiment of independent control.

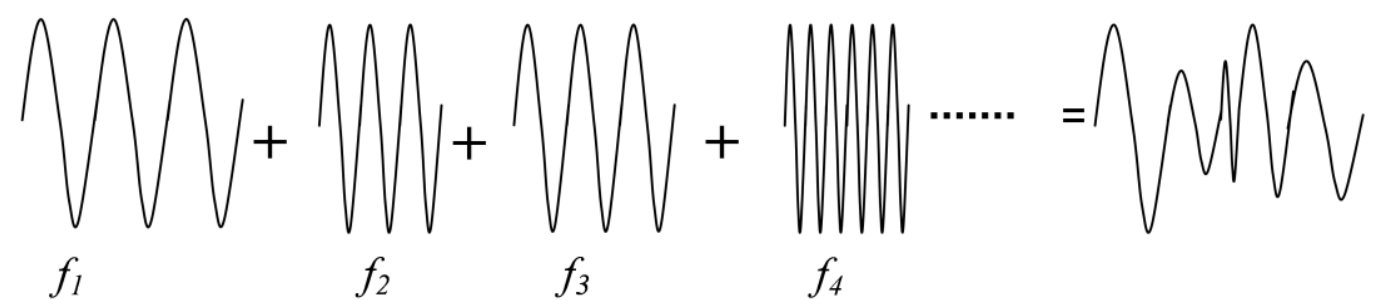

Fig.3 Multi-valve driving method 1: superimposing method which combines many frequencies

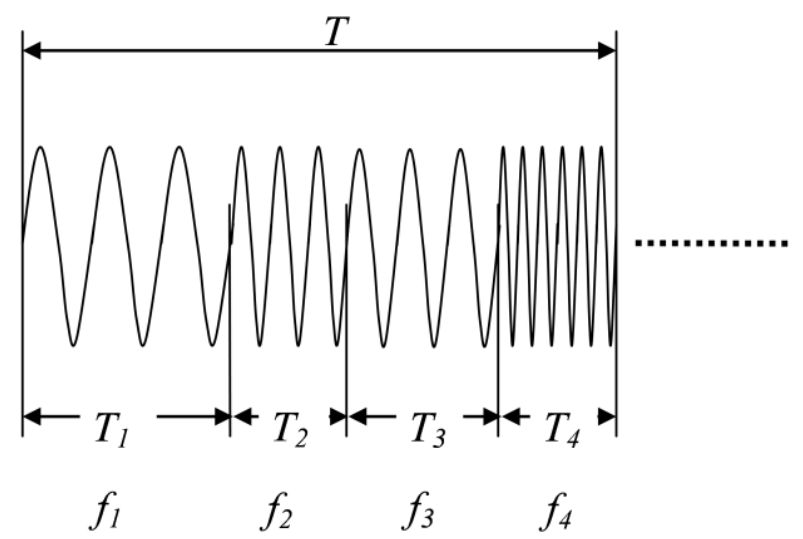

Fig.4 Multi-valve driving method 2: time-sharing method which connects many frequencies serially

\section{Design and fabrication of the prototype valve}

The primitive valve shown in Fig.2 doesn't work well because of friction between the vibrators and the case [10]. In this report, we have designed, developed and used a new prototype valve as shown in Fig.5, which consists of a rubber button valve, two vibrators and springs. This valve is configured with two mass-spring systems: the active $m-k$ system, which is driven by the air vibration in a air supply line and the passive $m-k$ system, which is driven passively by the active $m-k$ system. 
A rubber bellows-metal spring combination is used for the active $m-k$ system and a metal spring is used for the passive $m-k$ system. The driving air line is connected to the active rubber bellows and also to the input of the valving element, which is called the rubber button valve [15]. The rubber button valve realizes on/off function by the pushing force, like a mechanical button and solves the problem of the friction of vibrating objects. The rubber button valve is manufactured by a simple method, shown in Fig. 6. The first step of the manufacturing process is deciding on the design by using the nonlinear finite element method (FEM). Second, a mold based on the nonlinear FEM result is fabricated by CAD/CAM. Finally, the rubber button valve is molded and bonded by excimer surface important processing. The operation of the prototype valve as an on/off valve was confirmed in a step response experiment.

The resonant frequency of the prototype valve is designed by configuring the mass and spring constants in the mass-spring systems. The maximum frequency of the high-speed pneumatic valve generating air vibrating as the oscillator in the experimental system is approximately $50[\mathrm{~Hz}]$. Therefore, the four different resonant frequencies of the four prototype valves have to be less than 50 $[\mathrm{Hz}]$. The mass is configured by changing the material and volume. The materials are aluminum, stainless steel, copper, and polyacetal. The parameters of the four prototype valves are shown in Table 1. The theoretical resonant frequencies are shown in Table 1 the resonant frequencies when the vibrators are in contacting as calculated by Eq. (4). 


$$
f_{n}=\sqrt{\frac{\left(k_{1}+k_{2}\right)}{\left(m_{1}+m_{2}\right)}}
$$

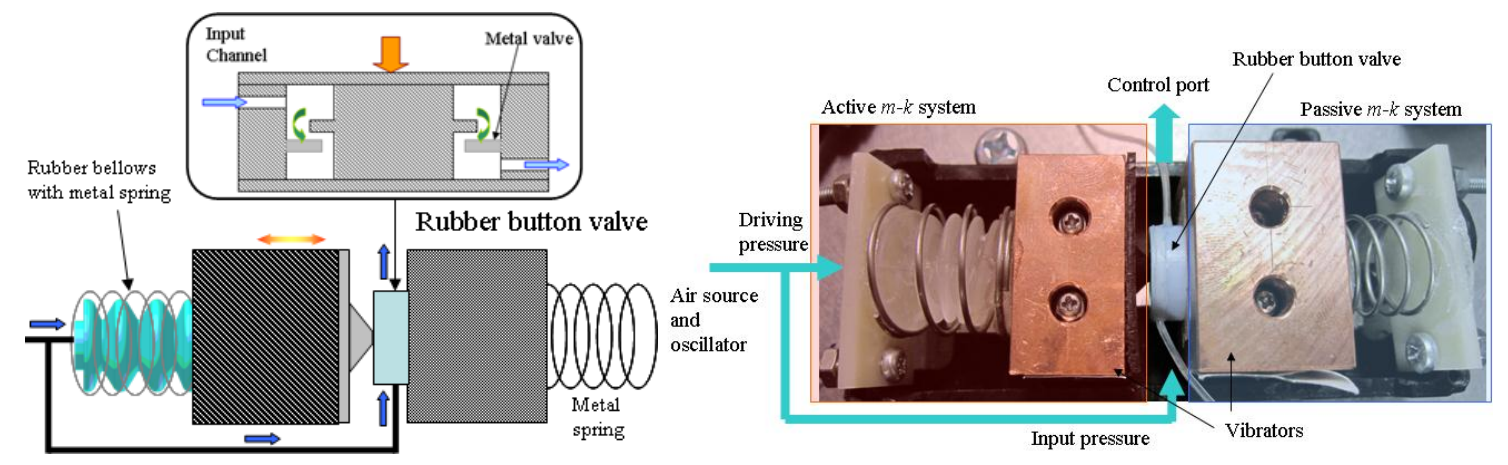

Fig.5 Prototype valve (left: inside view; right: structure as an on/off valve)

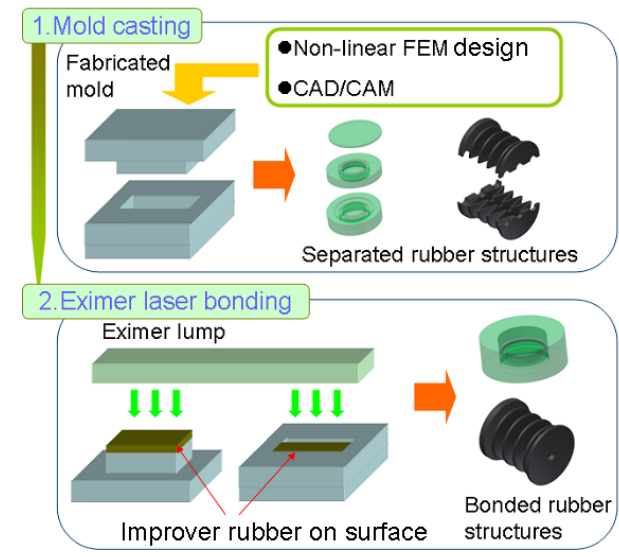

Fig.6 Manufacturing method for the rubber button valve [15]

Table 1 Parameters of the developed valves

\begin{tabular}{|c|c|c|c|c|c|c|}
\hline \multirow{2}{*}{ Valve } & \multirow{2}{*}{$\begin{array}{l}\text { Material of } \\
\text { vibrators }\end{array}$} & \multicolumn{2}{|c|}{ Active $m-k$ system } & \multicolumn{2}{|c|}{$\begin{array}{c}\text { Passive } m-k \\
\text { system }\end{array}$} & \multirow{2}{*}{$\begin{array}{c}\begin{array}{c}\text { Resonant } \\
\text { frequency }\end{array} \\
f_{n}(\mathrm{~Hz})\end{array}$} \\
\hline & & $m_{l}(\mathrm{~g})$ & $k_{l}(\mathrm{~N} / \mathrm{mm})$ & $m_{2}(\mathrm{~g})$ & $k_{2}(\mathrm{~N} / \mathrm{mm})$ & \\
\hline A & Cupper & 80.2 & 0.30 & 133.3 & 0.49 & 9.6 \\
\hline B & $\begin{array}{c}\text { Stainless } \\
\text { steel }\end{array}$ & 33.2 & 0.30 & 86.7 & 0.49 & 12.9 \\
\hline $\mathrm{C}$ & Polyacetal & 12.4 & 0.50 & 8.0 & 0.49 & 35.1 \\
\hline D & Aluminum & 23.9 & 0.30 & 25.2 & 0.50 & 20.3 \\
\hline
\end{tabular}




\section{Experimental evaluations of the resonant valve}

\subsection{Resonant motion of the vibrators}

The evaluation system for the resonant motion of the four prototype valves is shown in Fig. 7.

The motion of the two vibrators in the prototype valve is measured by a motion-capture system. The marker for capturing the motion is placed on the center of each vibrator. The frame rate of the high-speed camera for the motion capture is 150 [frame/s]. Sinusoidal pneumatic vibrations are applied to the valve. The four prototype valves are connected to the oscillator with one air line and are controlled from 5 [Hz] to 40 [Hz]. A high speed valve (FEST corp., MPYE-5-1/8LF-010-B) is used as the oscillator, which has the maximum response frequency of $100[\mathrm{~Hz}]$ and the maximum flow capacity of $350[1 / \mathrm{min}]$.

The experimental results of the motion of each vibrator are shown in Fig. 8. When the frequency is the non-resonant frequency, the vibrators keep contacting and the distance between the two vibrators is constant. The frequency having the largest distance between the two vibrators shows the resonant motion of the prototype valves. From the results, the use of the resonant frequency as an on/off valve was confirmed for the four different resonant frequencies. 


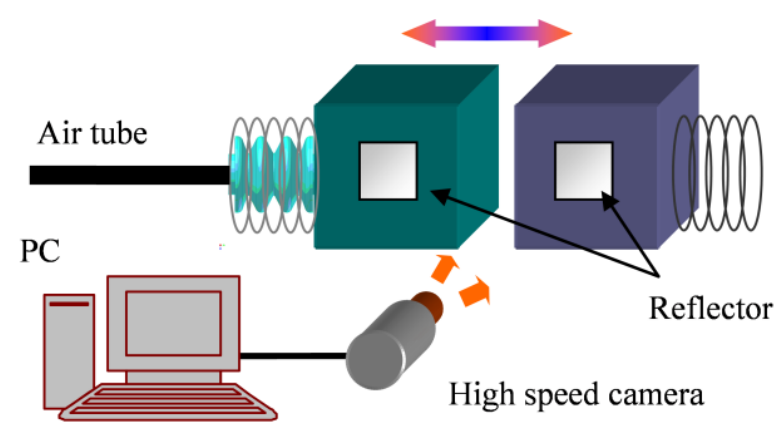

Fig.7 Motion capture system for measuring the resonant motion of the four prototype valves

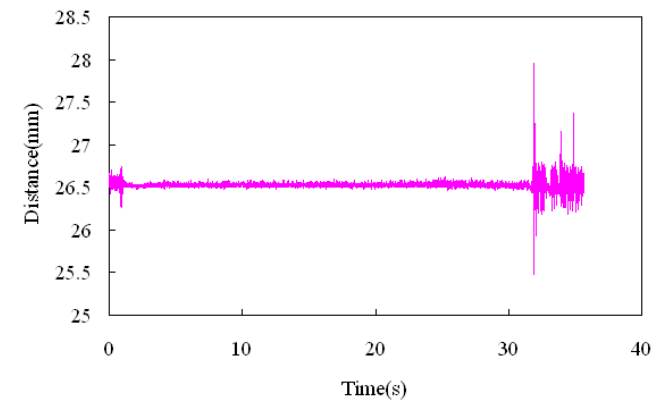

(a) motion of valve $\mathrm{A}$

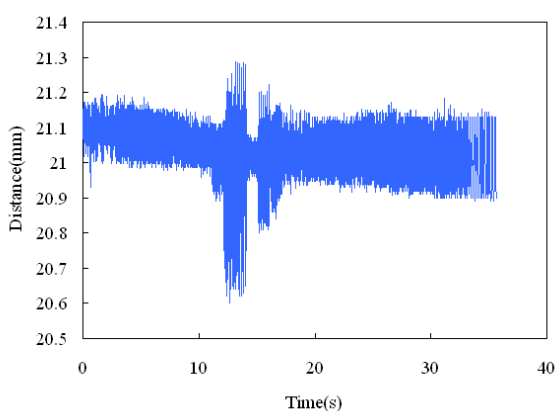

(c) motion of valve $\mathrm{C}$

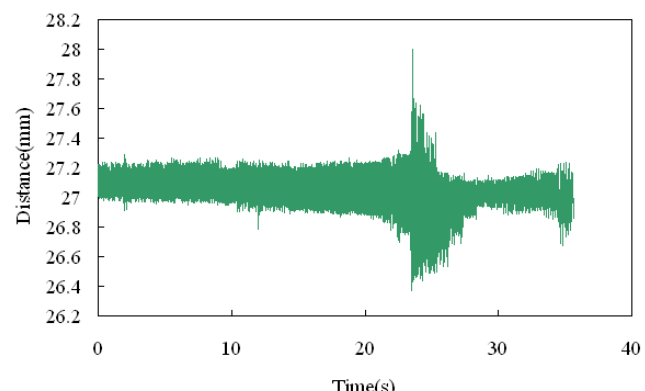

(b) motion of valve $\mathrm{B}$

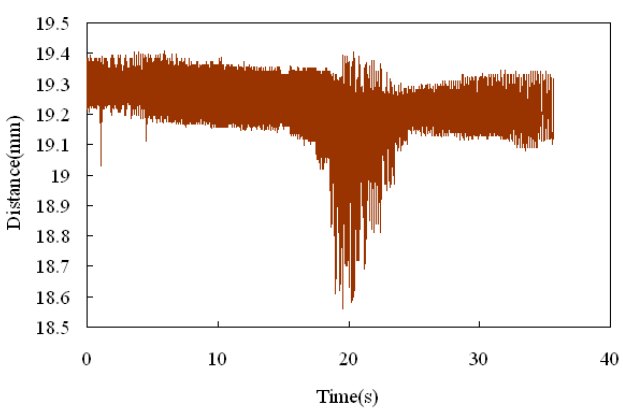

(d) motion of valve $\mathrm{D}$

Fig.8 Experimental results of the motion of the vibrators 


\subsection{Experiments of independent pneumatic control}

In the next experiment, the purpose is the confirmation of independent driving of the four prototype valves by the superimposing and time-sharing methods. The experimental system for the four prototype valves as pneumatic on/off valves is shown in Fig. 9. The parameters of this system are the same as those used in the evaluation system for motion capture. The output pressure from each prototype valve is measured by a pressure sensor. Since the rubber button valve has no exhausting function for the control port as shown in Fig.5. Small flow control are used as a leak valve as shown in Fig.9.

The experimental results of the pressure output for the frequency changes are shown in Fig. 10. The frequency is changed from $40[\mathrm{~Hz}]$ to $5[\mathrm{~Hz}]$ by $1[\mathrm{~Hz}]$ every $3[\mathrm{~s}]$. The standard signal is a sinusoidal wave, as shown in Eq. (1). For each valve, a sharp change of the pressure value was confirmed. Figure 11 shows the experimental results of the output pressure due to the changing amplitude of the signal. The output pressure changes for the different amplitude ratio in each valve. The amplitude ratio is defined the amplitude voltage divided by the max amplitude voltage. Figure 12 shows the experimental results of the step response for the resonant frequency of valve A. This one valve is driven only by the resonant frequency, and the other valves are in the off stage due their non-resonant frequencies. The single driving in the case of other valves was also confirmed. 
Figure 13 shows the results of multi-valve driving by two-valve driving, three-valve driving, and four-valve driving for each method. In the superimposing method, two-valve driving is successful, as shown on the left side of Fig. 13 (a). However, three-valve and four-valve drives are not successful, as shown on the left side of Fig. 13 (b) and (c). In contrast, in the time-sharing method, multi-valve driving is successful, as shown on the right of Fig. 13 (a)-(c). The output pressures corresponding to each resonant frequency are confirmed in each figure. The output pressure vibrates comparison with the single-driving. This vibrating error can possibly be reduced by optimizing the cycle time of each frequency $\left(T_{1}, T_{2}, T_{3}\right.$, and $T_{4}$ in Fig. 4). The different waveforms result from the time constant of each valve. However, the output pressures can be controlled by changing the amplitude of each signal.

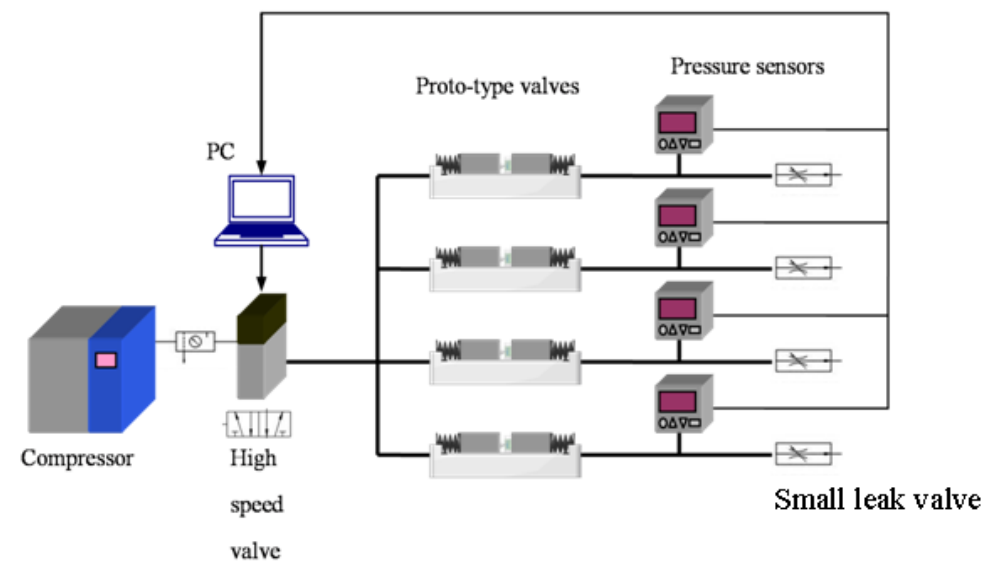

Fig.9 Independent control system of four prototype valves 


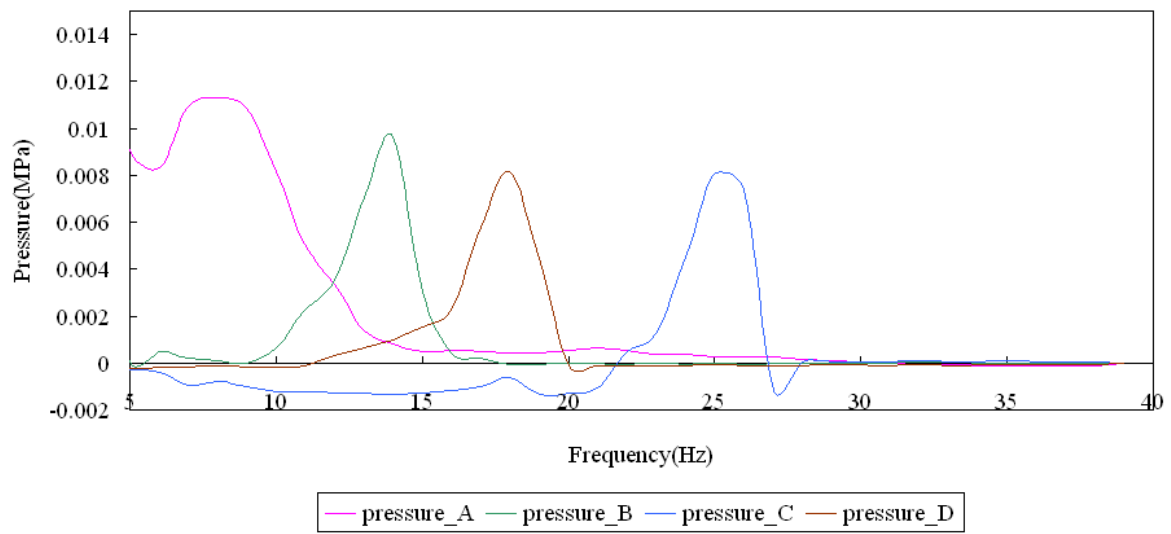

Fig.10 Experimental results of output pressure relative to the frequency response

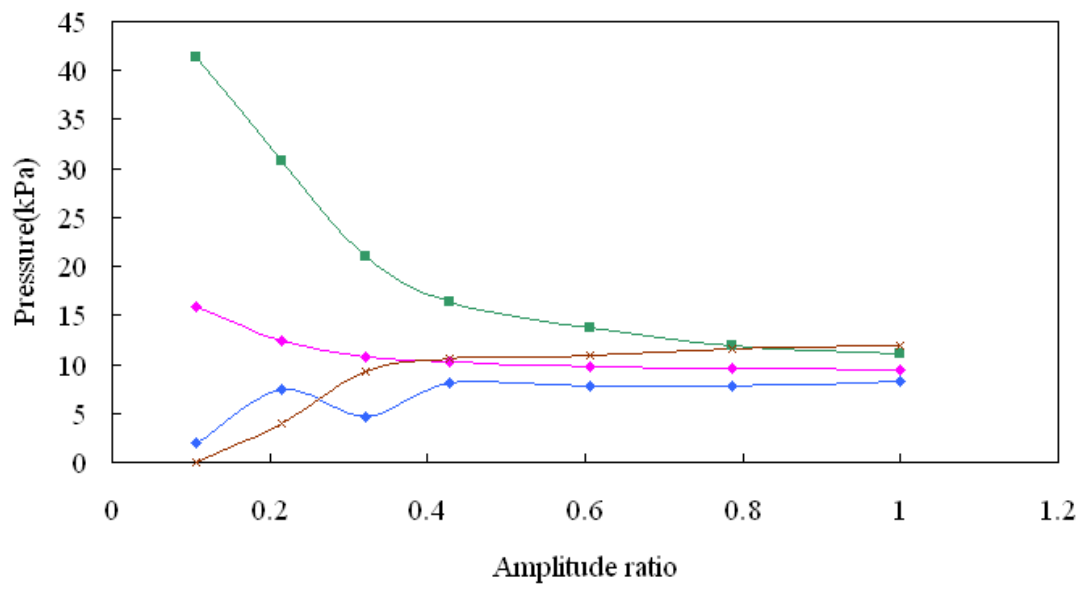

Fig.11 Experimental results of the output pressure control relative to the change of amplitude

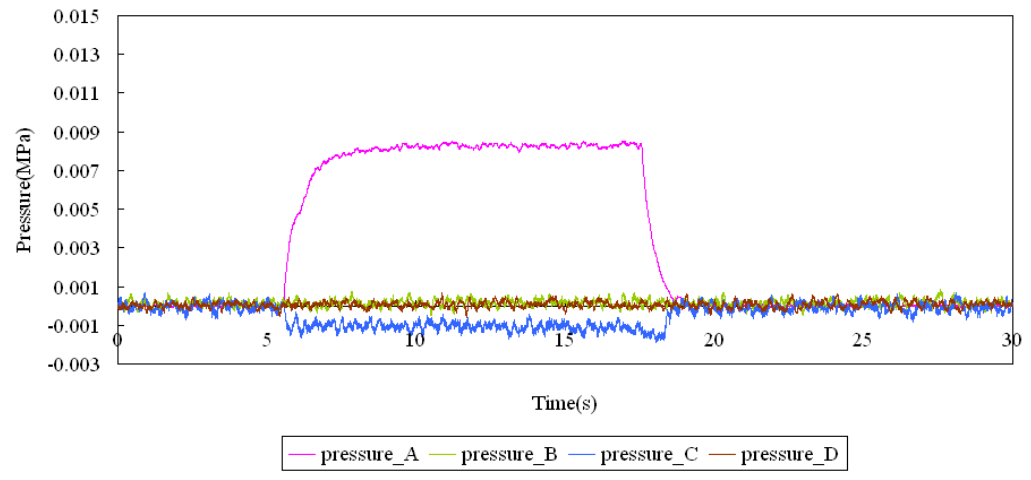

Fig.12 Step response for single-valve driving, valve A 

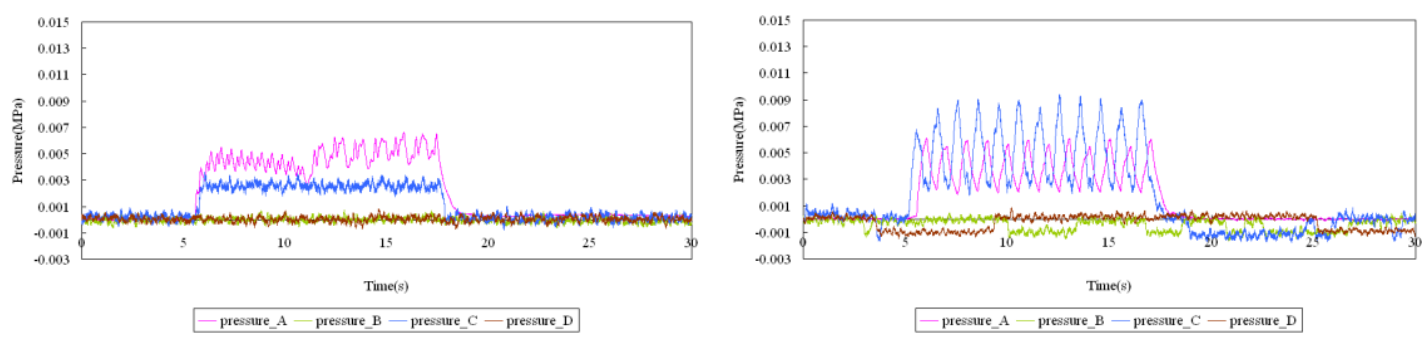

(a) two-valve driving, valves $\mathrm{A}$ and $\mathrm{C}$
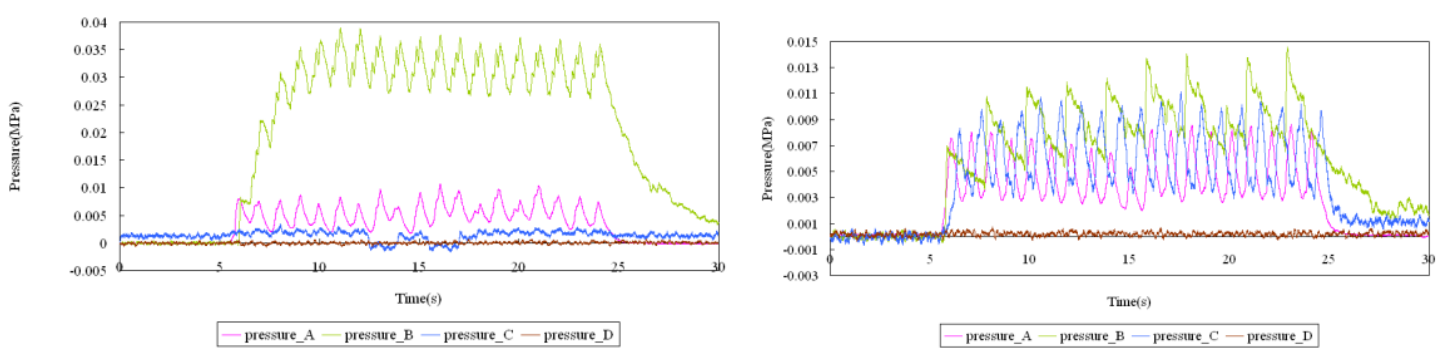

(b) three valve driving, valves A, B and C
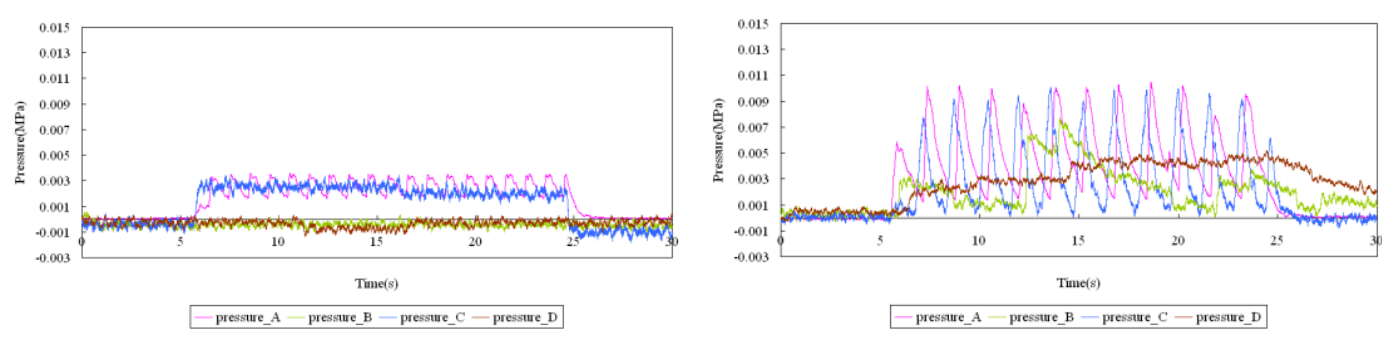

(c) four-valve driving

Fig.13 Comparison of the experimental results for the superimposing method (left) and time-sharing method (right) for multi-valve driving

\section{Conclusion}

In this research, a new pneumatic control method for multi-DOF pneumatic systems that achieves downsizing of the pneumatic valves and the entire system is proposed. The multiplex pneumatic 
transmission consists of the resonant valve, the oscillator, and air tube only. Thus, this system offers independent driving for multi-pneumatic actuators by the air vibration in one air line.

A prototype valve was developed for confirming expected operation. A new prototype valve is developed for this system. A key point of the prototype valve is a valving element which is the rubber button valve. The rubber button valve is manufactured by a simple process of rubber molding and excimer bonding. Four prototype valves having different resonant frequencies were developed and used in evaluation experiments.

The resonant motion of the vibrators was confirmed by a motion-capture system. Thus, single-valve driving was confirmed by a frequency response experiment and the resonant frequencies of the four prototype valves were clarified. A multi-valve driving method called the time-sharing method is also proposed for driving several valves at the same time. This method was compared with the superimposing method, proposed in a previous paper. The superimposing method is a multiplex method that superimposes many frequencies. In contrast, the time-sharing method connects several resonant frequencies serially in a short time. In the experiment using the time-sharing method, multiplex driving of the four valves was confirmed. In future tasks, optimization of the cycle time of each frequency will be investigated in the time-sharing method.

The resonant valves achieved driving as on/off valves without an electric wire by only air supply line. The structure of the resonant valve is effective for achieving multiplex pneumatic transmission. 
In addition, the time-sharing method for independent driving is effective for multi-pneumatic drive systems. One problem in the current prototype system is in the response of the oscillator and valves. As a future work we are challenging a new system using piezp vibrators and new valves, which will realize higher bandwidth of this system and will make the period of sinusoidal wave imposing shorter.

\section{Acknowledgments}

This research was supported by a Grant-in-Aid for Scientific Research in Priority Areas (No. 438) "Intelligent Actuators for Multi-Degrees-of-Freedom Mechatronics (16078209)" and a Grant-in-Aid for JSPS Fellows from the Ministry of Education, Culture, Sports, Science and Technology of Japan ("Research of pneumatic mechatronics having multi-degrees of freedom by multiplex pneumatic transmission").

\section{References}

1. K. Kawashima, “A Tendency at Pneumatic Areas in 2008," Journal of the Japan Fluid Power System Society, Vol. 40, No. E11, 2008 (in Japanese). 
2. M. Kosugi, "The Technology for Reducing Electric Wire of the Pneumatic Valve, and the State of Field Network about Pneumatic Devices,” Journal of the Japan Fluid Power System Society, Vol. 38, No. 3, 41-44, 2007 (in Japanese).

3. J. A. Riofrio and E. J. Barth, "Design of a Free Piston Pneumatic Compressor as a Mobile Robot Power Supply," In Proceedings of the 2005 IEEE International Conference on Robotics and Automation, pp. 235-240, Barcelona, Spain, 2005.

4. Y. Maeda, T. Noritsugu, D. Sasaki, K. Takano, T. Okamoto and S. Sato, "Development of Small-sized Air Pump using Balloon Vibrator for Wearable Devices," editorial committee of Seventh JFPS International Symposium on Fluid Power, P2-27, pp. 737-742, 2008.

5. P. Post, V. Zoppig and K. Neumann, "Fast, Small, Precise, and Efficient: Matching the Requirements on Modern Industrial Pneumatics," ACTUATOR 2008 11th International Conference on New Actuator, A4.0, pp. 141-144, 2008.

6. T. Akagi, S. Dohta and S. Katayama, "Development of Small-sized Flexible Control Valve Using Vibration Motor," editorial committee of Seventh JFPS International Symposium on Fluid Power, P2-25, pp. 725-730, 2008.

7. S. Yun, K. Lee, H. Kim and H. So, "Development of the Pneumatic Valve with Bimorph Type PZT Actuator," MATERIALS CHEMISTRY AND PHYSICS 97, 1-4, 2006. 
8. S. Jien, Y. Ogawa, S. Hirai and K. Honda, "Performance Evaluation of a Miniaturized Unconstrained Digital On-Off Switching Valve," In Proceedings of the 2008 IEEE/ASME International Conference on Advanced Intelligent Mechatronics, pp. 659-664, 2008.

9. K. Suzumori, Y. Nishioka and T. Kanda, "Pneumatic Actuator System Operated by Multiplex Pneumatic Transmission (1st report; verification of working principle)," In Proceedings on Autumn Conference of Japan Fluid Power System Society 2006, 4, 2006 (in Japanese).

10. Y. Nishioka and K. Suzumori, "Multiplex Pneumatic Transmission Realizing the Wireless of the Valve and the Tubeless," Hydraulics and Pneumatics, 2009 (in Japanese).

11. A. Kitagawa, S. Jing, C. Liu and H. Tsukagoshi, "A Study on a Sound Operated Valve for a Wearable Pneumatic System, “ In Proceedings of the 7th JFPS International Symposium on Fluid, P1-33, pp. 433-438, 2008.

12. K. Ikuta, H. Ichikawa, K. Suzuki and D. Yajima, "Multi-degree of Freedom Hydraulic Pressure Driven Safety Active Catheter," In Proceedings of the 2006 IEEE International Conference on Robotics and Automation, pp. 4161-4166, 2006.

13. Y. Nishioka, K. Suzumori, T. Kanda and S. Wakimoto, "Pneumatic Valve Operated Multiplex Pneumatic Transmission," Journal of Advanced Mechanical Design, Systems, and Manufacturing, Special Issue on 2007-Manufacturing, Mechanical Design and Tribology, Vol.

2, No. 2, April, 2008. 
14. Y. Nishioka, K. Szumori, T. Kanda and S. Wakimoto, "A New Pneumatic Control System Using Multiplex Pneumatic Transmission," editorial committee of Seventh JFPS International Symposium on Fluid Power, P1-34, 2008.

15. Y. Nishioka, K. Suzumori, S. Wakimoto, T. Kanda and K. Ogura, "Rubber Button Valve and Bellows Realizing Resonant Valve for Multiplex Pneumatic Control," In Proceedings of the ISRM 2009, IFToMM 1. International Symposium on Robotics and Mechatronics, 32, 2009.

Biography

Yasutaka Nishioka was born in 1984. He received the B. Eng. and the M. Eng. degrees in system engineering from the Okayama University, Japan in 2006, and 2008, respectively. He has been a doctoral student of the Okayama University since 2008. His research interests are pneumatic actors and flexible devices.

He is a Research Fellow of the Japan Society for the Promotion of Science from 2009 to 2011.

$\mathrm{He}$ is a member of the Japan Society of Mechanical Engineers, and a student member of the Robotics Society of Japan and the Japan Fluied Power System Society. 
Koichi Suzumori was born in 1959. He received a Doctor Degree from Yokohama National University in 1990. He worked for the Toshiba R\&D Center from 1984 to 2001, and for the Micromachine Center, Tokyo from 1999 to 2001 . He has been a professor at Okayama University, Japan since 2001.

He is a member of the Japan Society of Mechanical Engineers, the Robotics Society of Japan, IEEE and the Institute of Electrical Engineers of Japan.

Takefumi Kanda was born in Fukuoka, Japan, on June 18, 1972. He received the B. Eng., the M. Eng. and the Dr. Eng. degrees in precision machinery engineering from the University of Tokyo, Japan in 1997, 1999, and 2002, respectively.

From 2002, he was a research associate and lecturer at Okayama University, Japan. Since 2007, he has been an associate professor at Okayama University. His research interests are micro sensors, micro actuators, micro systems and piezoelectric film.

He is a member of the Japan Society for Precision Engineering, the Institute of Electrical Engineers of Japan, IEEE, the Japan Society of Mechanical Engineers and the Robotics Society of Japan.

Shuichi Wakimoto received BE, ME and DE degrees from Okayama University, Japan in 2002, 2004 and 2007, respectively. He was a Research Fellow of the Japan Society for the Promotion of 
Science from 2004 to 2007. From 2007 to 2009, he was an Assistant Professor at the Graduate School of Natural Science and Technology, Okayama University, Japan. Since 2009, he has been an Assistant Professor at Research Core for Interdisciplinary Sciences, Okayama University, Japan. His research interests are soft mechanics including flexible sensors and actuators. He is a member of the Japan Society of Mechanical Engineers (JSME) and the Robotics Society of Japan (RSJ). 